\title{
Electronic Structure and Magnetism in Transition Metals Doped 8-Hydroxy-Quinoline Aluminum
}

\author{
Jeong Min Baik, ${ }^{\dagger}$ Yoon Shon, ${ }^{\ddagger}$ Seung Joo Lee, ${ }^{\ddagger}$ Yoon Hee Jeong, ${ }^{\S}$ Tae Won Kang, ${ }^{\ddagger}$ and \\ Jong-Lam Lee*,† \\ Department of Materials Science and Engineering, Pohang University of Science and Technology (POSTECH), \\ Pohang, Kyungbuk 790-784, Korea, Quantum Functional Semiconductor Research Center, Dongguk University, \\ 3-26 Pil-dong, Chung-ku, Seoul 100-715, Korea, and Department of Physics, Pohang University of Science and \\ Technology (POSTECH), Pohang, Kyungbuk 790-784, Korea
}

Received July 18, 2008; E-mail: jllee@ postech.ac.kr

Ferromagnetic semiconductors with a high Curie temperature $(>300 \mathrm{~K})$ are believed to be suitable for future spintronics devices such as spin-light emitting diodes, spin-field effect transistors, and quantum computers. ${ }^{1,2}$ The advantages of these devices include increased processing speed, lower power consumption, and higher information density, compared with conventional devices. ${ }^{2}$ The recent recovery of organic semiconductors with magnetically aligned spins is also interesting because they can be synthesized under much less stringent conditions than inorganic semiconductors due to the low-temperature processing and mechanical flexibility. In particular, $\pi$-conjugated organic semiconductors such as 8-hydroxy-quinoline aluminum ( $\mathrm{Alq}_{3}$, inset of Figure 1a) may be very good for transport of spin-polarized carriers because of the long spin diffusion length and giant magnetoresistance. ${ }^{3}$ However, the spin injection efficiency was too low to be used in spin devices and the operation temperature was below $300 \mathrm{~K}$, which might be attributed to the formation of insulating complexes at the interface of metal/Alq $\mathrm{Al}_{3}$ (i.e., the interface roughness increased, causing suppression of spin injection to $\mathrm{Alq}_{3}$ layer) and the conductivity mismatch. $^{4}$

Here we address this low spin injection efficiency issue for $\mathrm{Alq}_{3}$ and suggest a solution. First let us note that both problems may be simultaneously solved by using organic-based magnets. Tetracyanoethylene-based organic magnets, for example, have been reported to be ferromagnetic at low and even at room temperatures. ${ }^{5}$ Thus we attempted to modify $\mathrm{Alq}_{3}$ itself and make it magnetic; $\mathrm{Alq}_{3}-$ based magnets, if they possess ferromagnetism at room temperature, would be an ideal spin injection layer to an original semiconductor $\mathrm{Alq}_{3}$.

A glass coating with $150 \mathrm{~nm}$ of indium-tin oxide (ITO) was used as the starting substrate. The ITO samples (after a general cleaning process) were loaded into a thermal evaporator at $1.0 \times$ $10^{-6}$ Torr, on which Co-doped $\mathrm{Alq}_{3}$ layers with a thickness of 100 $\mathrm{nm}$ were coevaporated of pure Co metal (99.99\%) and $\mathrm{Alq}_{3}$ powders at room temperature. The Co concentrations in $\mathrm{Alq}_{3}$ were determined to be $5 \%(\mathrm{Co} / \mathrm{Al}$ atomic ratio $=0.5)$ and $10 \%(1)$ using the thickness ratio of $\mathrm{Co}$ and $\mathrm{Alq}_{3}$. Ni-doped $\mathrm{Alq}_{3}$ was also prepared by the same method. For the measurement of magnetic properties, a Ag layer was deposited on the organic layers, playing a role in protecting the organic layers from moisture. Magnetization measurement was carried out using a superconducting quantum interference device magnetometer (MPMSXL, Quantum Design Co., Ltd.). We also employed a variety of measurement techniques utilizing synchrotron radiation, including X-ray absorption spectroscopy

\footnotetext{
† Department of Materials Science and Engineering, POSTECH.

* Dongguk University.

$\$$ Department of Physics, POSTECH.
}
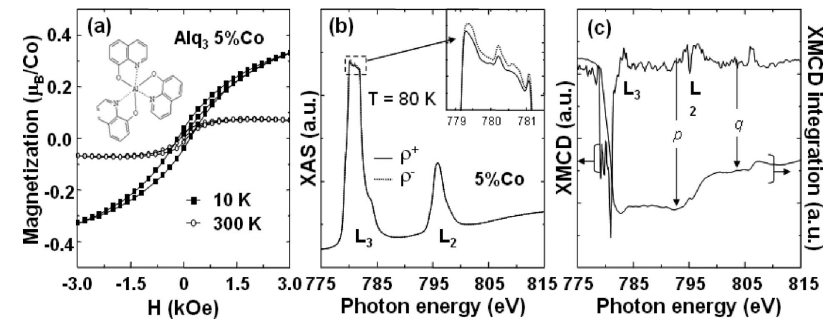

Figure 1. (a) Magnetization curves of 5\% Co-doped $\mathrm{Alq}_{3}$ at 10 and 300 K. (b) XAS and (c) XMCD spectra and its integrated value at the Co $L_{2}, L_{3}$ edges of $5 \%$ Co-doped $\mathrm{Alq}_{3}$ sample. The photon spin was aligned parallel $\rho^{+}$(solid line) and antiparallel $\rho^{-}$(dotted line) to the applied magnetic field, respectively; the difference between the absorption spectra is shown in the inset.

(XAS), and X-ray magnetic circular dichroism (XMCD) to elucidate the origin of the ferromagnetism in the compound.

Figure 1a shows the magnetic curves for the 5\% Co-doped $\mathrm{Alq}_{3}$ at 10 and $300 \mathrm{~K}$. The hysteresis loop of the sample at $10 \mathrm{~K}$ showed clear ferromagnetic behavior with a coercive field of 170 Oe and a magnetic moment of $\sim 0.33 \mu_{\mathrm{B}} / \mathrm{Co}$ at $0.3 \mathrm{~T}$. From the magnetic curve at $300 \mathrm{~K}$ and the temperature dependence of the magnetic moment (Supporting Information Figure S1), it is obvious that the ferromagnetic features are still maintained up to $300 \mathrm{~K}$, meaning that the Curie point $T_{\mathrm{C}}$ is higher than $300 \mathrm{~K}$. Paramagnetic characters probably due to isolated Co ions, however, are also observed at low temperatures below $\sim 70 \mathrm{~K}^{6}{ }^{6}$ One obvious possibility that the observed ferromagnetic properties are due to the formation of Co-related precipitates must be checked. In fact, we could observe the enhanced paramagnetic properties as the $\mathrm{Ni}$ concentration in $\mathrm{Alq}_{3}$ increased (Supporting Information Figure S2).

Figure $1 \mathrm{~b}$ shows the Co $2 p$ XAS spectra for $5 \%$-doped $\mathrm{Alq}_{3}$. The peaks shown in the XAS spectra correspond to transitions from the $2 p_{3 / 2}\left(L_{3}\right.$ edge) and $2 p_{1 / 2}\left(L_{2}\right.$ edge) states to the Co $3 d$ state. The spectral shape is quite different from typical metallic spectra, and it can be recognized that the Co state is almost the same as that for $\mathrm{Co}^{2+}$ ions surrounded by oxygen atoms. ${ }^{7}$ The difference between the absorption spectra measured with the two opposite signs of polarization was obtained between the opposite directions of magnetization, traveling parallel $\left(\rho^{+}\right)$and antiparallel $\left(\rho^{-}\right)$to the direction of the circularly polarized X-ray wave vector and was plotted in Figure 1c. It clearly shows XMCD peaks $\left(\mu_{+}-\mu_{-}\right)$with a negative sign at the $L_{3}$ edge. It should be emphasized that the XMCD spectra also show a multiplet structure, unlike those of Co metal. ${ }^{8}$ The integrated value of the XMCD spectrum is also shown in the figure. The orbital magnetic moment contribution versus the spin/dipole magnetic moment can be expressed as $m_{\mathrm{L}} /\left(m_{\mathrm{S}}+7 m_{\mathrm{T}}\right)=2 q /(9 p-6 q),{ }^{9}$ 


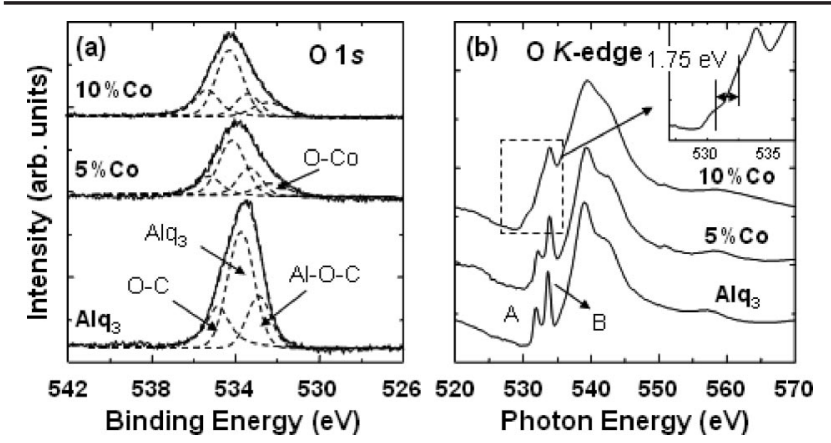

Figure 2. (a) SRPES spectra of O $1 s$ core level for Co-doped $\mathrm{Alq}_{3}$ with Co concentration. (b) NEXAFS spectra at the $\mathrm{O} K$-edge of Co-doped $\mathrm{Alq}_{3}$ with different Co concentrations.

and the value is calculated to be 0.33 , much larger than that of the bulk Co (0.09). ${ }^{8}$ Here, $q$ and $p$ are the integrated value over the whole $h v$ range $\left(L_{3}+L_{2}\right)$ and the integrated XMCD only in the $L_{3}$ region, respectively. This indicates that the magnetism in the present sample is attributed to Co ions with localized $3 \mathrm{~d}$ electrons, not Co metal.

Figure 2a displays the SRPES of O $1 s$ core levels with Co concentration. For as-deposited $\mathrm{Alq}_{3}$, the $\mathrm{O} 1 s$ spectrum consisted of three components, such as the $\mathrm{Al}-\mathrm{O}-\mathrm{C}$ bond $(532.9 \mathrm{eV}), \mathrm{Alq}_{3}$ $(533.75 \mathrm{eV})$, and the $\mathrm{O}=\mathrm{C}$ bond $(534.85 \mathrm{eV})$. When $\mathrm{Co}$ was doped, the spectrum showed asymmetry at the lower bonding energy of the $\mathrm{Al}-\mathrm{O}-\mathrm{C}$ bond and the full width at half-maximum value increased from 1.79 to $2.10 \mathrm{eV}$ (10\% sample). This means that an additional bonding is superimposed in the $\mathrm{O} 1 s$ spectra. The new peak could be separated at the lower bonding energy by $1.0 \mathrm{eV}$ relative to the peak of the $\mathrm{Al}-\mathrm{O}-\mathrm{C}$ bond, in good agreement with that of the $\mathrm{O}-\mathrm{Co}$ bond. ${ }^{10}$ The NEXAFS spectra at the $\mathrm{O} K$-edge (Figure $2 b$ ) shows the formation of a new state due to the chemical reaction. For as-deposited $\mathrm{Alq}_{3}$, the spectra have structures $\mathrm{A}$ and $\mathrm{B}$, corresponding to transitions from the $1 s$ orbital to the low unoccupied molecular orbital (LUMO) and LUMO+1, respectively. ${ }^{11}$ When Co was doped, the structures become weak and one new peak emerged at the lower energy by $1.75 \mathrm{eV}$ relative to peak A. The occupancy of the LUMO will decrease the transition intensity from the core levels, meaning that the doped Co provides electrons for the $\mathrm{Alq}_{3}$. This can be also explained by the UPS spectra and UV absorption spectra (Supporting Information Figure S3).

The formation of a new state in the forbidden energy gap of $1.75 \mathrm{eV}$ below the LUMO level and the shift to a higher binding energy of the entire molecule orbital give evidence that the Co doping to $\mathrm{Alq}_{3}$, rather than a nanosized Co cluster, can produce electron trap sites. This can be expected by the high reactivity between metals and $\mathrm{Alq}_{3}$, even at room temperature. ${ }^{12}$ Thus, a reasonable explanation for the magnetism may be possible by the bound magnetic polarons model, in which defects (by impurities) can play an important role in the system. ${ }^{13}$ In fact, the charge transferred to the $\mathrm{Alq}_{3}$ was known to be localized on the pyridyl side of the quinolate ligand, resulting in the formation of polarontype states. ${ }^{11}$ The microstructural analysis does not show the formation of magnetic clusters such as Co-oxides and metallic Co (Supporting Information Figure S1). Moreover, $\mathrm{CoO}$ is an antiferromagnetic material, whose Néel temperature is $293 \mathrm{~K} .{ }^{14}$ Metallic
Co can be also easily excluded because the valence band maximum was separated at the higher binding energy by $\sim 1.3 \mathrm{eV}$ relative to the Fermi level, and the saturation moment $\left(0.33 \mu_{\mathrm{B}} / \mathrm{Co}\right.$ atom $)$ was very low compared to $3.0 \mu_{\mathrm{B}} / \mathrm{Co}$ atom of metallic Co. ${ }^{15}$ These results confirm that the ferromagnetic property is an intrinsic property of Co-doped $\mathrm{Alq}_{3}$.

In conclusion, we report the room-temperature ferromagnetism in Co-doped 8-hydroxy-quinoline aluminum synthesized by thermal coevaporation of pure $\mathrm{Co}$ metal and $\mathrm{Alq}_{3}$ powders. A maximum magnetization of $\sim 0.33 \mu_{\mathrm{B}} / \mathrm{Co}$ at $0.3 \mathrm{~T}$ is obtained in $5 \%$ Co-doped $\mathrm{Alq}_{3}$. The $\mathrm{Co}$ atoms seem to interact with $\mathrm{O}$ atoms, providing electrons to $\mathrm{Alq}_{3}$ and producing new states which act as trap sites for electrons, localized on the quinolate ligand. Thus, the observed ferromagnetism can be explained by the bound magnetic polarons, due to the strong interaction of Co atoms and the $\mathrm{Alq}_{3}$ molecule.

Acknowledgment. This work was supported in part by the Korean Research Foundation Grant funded by the Korean Government (MOEHRD) (KRF-2005-005-J13102) and in part by the Korea Science and Engineering Foundation (KOSEF) grant funded by the Korea government (MEST) (No. R-17-2008-024-01000-0). XMCD and SRPES using synchrotron radiation were carried out, respectively, at the $2 \mathrm{~A} 1$ and $4 \mathrm{~B} 1$ beamlines at Pohang Accelerator Laboratory.

Supporting Information Available: Experimental details and three figures. This material is available free of charge via the Internet at http:// pubs.acs.org.

\section{References}

(1) Krusin-Elbaum, L.; Newns, D. M.; Zeng, H.; Derycke, V.; Sun, J. Z.; Sandstrom, R. Nature 2004, 431, 672-676.

(2) Wolf, S. A.; Awschalom, D. D.; Buhrman, R. A.; Daughton, J. M.; von Molnár, S.; Roukes, M. L.; Chtchelkanova, A. Y.; Treger, D. M. Science 2001, 294, 1488-1495.

(3) (a) Xiong, Z. H.; Wu, D.; Vardeny, Z. V.; Shi, J. Nature 2004, 427, 821824. (b) Tanabe, S; Miwa, S; Mizuguchi, M; Shinjo, T; Suzuki, Y; Shiraishi, M Appl. Phys. Lett. 2007, 91, 063213 .

(4) (a) Yu, Z. G.; Berding, M. A.; Krishnamurthy, S. Phys. Rev. B 2005, 71, 060408. (b) Ren, J. F.; Fu, J. Y.; Liu, D. S.; Mei, L. M.; Xie, S. J. Synth. Met. 2005, 155, 611 .

(5) (a) Miller, J. S.; Calabrese, J. C.; Rommelmann, H.; Chittapeddi, S.; Zhang, J. H.; Reiff, W. M.; Epstein, A. J. J. Am. Chem. Soc. 1987, 109, 769-781. (b) Pokhodnya, K. I.; Epstein, A. J.; Miller, J. S. Adv. Mater. 2000, 12, 410-413.

(6) Alaria, J.; Bieber, H.; Colis, S.; Schmerber, G.; Dinia, A. Appl. Phys. Lett. 2006, $88,112503$.

(7) Kobayashi, M.; Ishida, Y.; Hwang, J. 1.; Mizokawa, T.; Fujimori, A.; Mamiya, K.; Okamoto, J.; Takeda, Y.; Okane, T.; Saitoh, Y.; Muramatsu, Y.; Tanaka, A.; Saeki, H.; Tabata, H.; Kawai, T. Phys. Rev. B 2005, 72, 201201.

(8) Chen, C. T.; Idzerda, Y. U.; Lin, H. J.; Smith, N. V.; Meigs, G.; Chaban, E.; Ho, G. H.; Pellegrin, E.; Sette, F. Phys. Rev. Lett. 1995, 75, 152-155.

(9) (a) Xie, T.; Kimura, A.; Qiao, S.; Moko, T.; Muro, T.; Taniguchi, M.; Pan, M.-H.; Jia, J.-F.; Xue, Q.-K. J. Phys.: Condens. Matter 2004, 16, S5783-S5786. (b) Thole, B. T.; Carra, P.; Sette, F.; van der Laan, G. Phys. Rev. Lett. 1992, 68, 1943-1946.

(10) Mouler, J. F.; Strickle, W. F.; Sobol, P. E.; Bomben, K. D. Handbook of X-Ray Photoelectron Spectroscopy; Perkin-Elmer: Eden Prairie, MN, 1992.

(11) Curioni, A.; Andreoni, W.; Treusch, R.; Himpsel, F. J.; Haskal, E.; Seidler, P.; Heske, C.; Kakar, S.; van Buuren, T.; Terminello, L. J. Appl. Phys. Lett. 1998, 72, 1575-1578.

(12) (a) Choong, V.-E.; Mason, M. G.; Tang, C. W.; Gao, Y. Appl. Phys. Lett. 1998, 72, 2689-2691. (b) Ding, H.; Gao, Y. Appl. Phys. Lett. 2005, 86, 213508.

(13) Wolff, P. A.; Bhatt, R. N.; Durst, A. C. J. Appl. Phys. 1996, 79, 51965198.

(14) Durst, A. C.; Bhatt, R. N.; Wolff, P. A. Phys. Rev. B 2002, 65, 235205.

(15) Ueda, K.; Tabata, H.; Kawai, T. Appl. Phys. Lett. 2001, 79, 988-990.

JA805276P 